\title{
Evolution of randomly perturbed Korteweg-de Vries solitons
}

\author{
Abdullaev, F. Kh.; Darmanyan, S. A.; Djumaev, M. R.; Majid, A. J.; Sørensen, Mads Peter
}

Published in:

Physical Review E. Statistical, Nonlinear, and Soft Matter Physics

Link to article, DOI:

10.1103/PhysRevE.52.3577

Publication date:

1995

Document Version

Publisher's PDF, also known as Version of record

Link back to DTU Orbit

Citation (APA):

Abdullaev, F. K., Darmanyan, S. A., Djumaev, M. R., Majid, A. J., \& Sørensen, M. P. (1995). Evolution of randomly perturbed Korteweg-de Vries solitons. Physical Review E. Statistical, Nonlinear, and Soft Matter Physics, 52(4), 3577-3583. https://doi.org/10.1103/PhysRevE.52.3577

\section{General rights}

Copyright and moral rights for the publications made accessible in the public portal are retained by the authors and/or other copyright owners and it is a condition of accessing publications that users recognise and abide by the legal requirements associated with these rights.

- Users may download and print one copy of any publication from the public portal for the purpose of private study or research.

- You may not further distribute the material or use it for any profit-making activity or commercial gain

- You may freely distribute the URL identifying the publication in the public portal 


\title{
Evolution of randomly perturbed Korteweg-de Vries solitons
}

\author{
F.Kh. Abdullaev, S.A. Darmanyan, and M.R. Djumaev \\ Theoretical Division of the Physical-Technical Institute, Uzbek Academy of Sciences, 700084, Tashkent-84, \\ G. Mavlyanova Str. 2-b, Uzbekistan \\ A.J. Majid and M.P. Sørensen \\ Institute of Mathematical Modelling, The Technical University of Denmark, Building 321, DK-2800 Lyngby, Denmark
}

(Received 30 December 1994; revised manuscript received 22 May 1995)

\begin{abstract}
The evolution of randomly modulated solitons in the Korteweg-de Vries (KdV) equation is investigated. The cases of multiplicative and additive noises are considered. The distribution function for the soliton parameters is found using the inverse scattering transform. It is shown that the distribution function has non-Gaussian form and that the most probable and the mean value of the soliton amplitudes are distinct. The analytical results agrees well with the results of the numerical simulations of the $\mathrm{KdV}$ equation with random initial conditions. The results obtained for the KdV equation is used to discuss the evolution of randomly modulated small-amplitude dark solitons in optical fibers and pulses in a nonlinear transmission lines.
\end{abstract}

PACS number(s): 02.50.-r, 05.40. $+\mathrm{j}, 52.35 . \mathrm{Mw}$

\section{INTRODUCTION}

The investigation of the evolution of randomly modulated pulses in nonlinear dispersive media represents an important problem for many branches of physics. A particular case of this problem is the propagation of nonlinear random waves in systems described by integrable or near-integrable equations [1-4]. Progress in this domain is achieved by the possibility of reducing the Cauchy problem for nonlinear partial differential equations to solving sequences of linear problems [5]. In the case of dispersionless nonlinear media described by the Burgers equation and its generalizations, the solution of the problem has been found by means of Cole-Hopf transformation. The Cole-Hopf transformation reduces the Burgers equation to the linear diffusion equation. This approach makes it possible to study the evolution of the random initial fields in order to find the spectrum of turbulence of waves excited by a random external sources and some related problems [6].

For nonlinear wave processes in a dispersive media the inverse scattering transform (IST) plays the role of the Cole-Hopf transformation [5]. To date, progress has been achieved for the analysis of presolitonic and near-solitonic initial conditions. The evolution of a random presolitonic initial wave in the framework of the IST was considered in [7] for the case of nonlinear Fraunhofer diffraction in a medium with Kerr-like nonlinearity. The near-solitonic initial condition is attractive both due to a relative simple analysis and because of wide application of solitons for the explanation of many physical phenomena. In [8] the Langmuir turbulence of soliton gas was studied for different modifications of the nonlinear Schrödinger equation (NLSE). It was shown that soliton states are the statistical attractors. This is another argument in favor of investigations of near-solitonic initial states.

The evolution of the near-solitonic fields in NLSE was studied in $[2,9]$ for different kinds of random initial modulations. Analogous problems for the sine-Gordon and Korteveg-de Vries (KdV) equations were investigated in $[1,4]$.

In $[2,4,9]$ the soliton parameter corrections caused by the random initial modulation of the soliton shape were calculated analytically. In this article we present the analytical and numerical calculations of the distribution function of the soliton parameters in the cases of multiplicative and additive noises. The analytical part of the calculations is based on the IST and cumulant method. The mean values, the standard deviation, and other statistical characteristics of soliton parameters are obtained. Good agreement of the analytical results with direct numerical calculations is demonstrated. The application of the results to the evolution of randomly modulated dark optical solitons and pulses in nonlinear transmission lines is discussed.

\section{FORMULATION OF THE PROBLEM: BASIC EQUATIONS}

Let us consider the problem of evolution of a randomly modulated initial soliton state in the $\mathrm{KdV}$ equation

$$
u_{t}-6 u u_{x}+u_{x x x}=0 \text {. }
$$

We will consider two models of random initial conditions: multiplicative and additive noises. The initial condition for the multiplicative noise is

$$
u_{0}(x)=u_{s}(x)[1+\epsilon(x)]=u_{s}(x)+\Delta u(x) .
$$

For the additive noise the initial condition is

$$
u_{0}(x)=u_{s}(x)+\epsilon(x) Q \operatorname{sech}^{2}(p x),
$$


where $u_{s}(x)=-2 q^{2} \operatorname{sech}^{2}(q x)$ is the single-soliton solution of the KdV equation. Below we will consider the case of broad noise, when $p \ll q$. For example, in experiments [10] the dynamics of dark solitons with 1-2 ps duration on a 200-ps-duration background pulse was investigated. So the value of the ratio $p / q$ for a randomly modulated background is $0.005-0.01$. In formulas (2.2) and (2.3) the function $\varepsilon(x)$ is the random Gaussian function with the properties

$$
\langle\varepsilon(x)\rangle=0,\langle\varepsilon(x) \varepsilon(y)\rangle=B(x-y) \ll 1 .
$$

Here angular brackets \langle\rangle denote averaging over all realizations of the random process $\varepsilon(x)$ and $B(x)$ is the correlation function of the noise.

According to the IST method for solution of the Cauchy problem (2.1) and (2.2) it is necessary to find the spectrum of the linear operator $\hat{L}$ associated with the $\mathrm{KdV}$ equation

$$
\hat{L} \psi=E \psi, E=k^{2}
$$

where

$$
\hat{L}=-\frac{d^{2}}{d x^{2}}+u_{0}(x)
$$

$u_{0}(x)$ is given by the expression (2.2). For $\varepsilon(x)=0$ it follows from (2.5) that there exists only one discrete level $E_{0}=-q^{2}$, with the corresponding eigenfunction

$$
\psi_{1}(x)=\sqrt{q / 2} \operatorname{sech}(q x) .
$$

The functions of the continuum spectrum are

$$
\psi_{K}(x)=\frac{1}{\sqrt{2 \pi}} \frac{K-i q \tanh (q x)}{K+i q} e^{i K x} .
$$

Below we shall consider $\Delta u(x)$ as a perturbation of the potential in the problem (2.5). Using the expressions (2.7) and (2.8) it is possible to represent the correction to the eigenvalue $\Delta E=E-E_{0}$ for the multiplicative noise (2.3) as a functional series of perturbation theory in power of a random process $\varepsilon(x)$

$$
\Delta E=\int_{-\infty}^{\infty} \varepsilon(x) a(x) d x+\int_{-\infty}^{\infty} \varepsilon(x) \varepsilon(y) b(x, y) d x d y+\cdots
$$

where $a(x)=-q^{3} \operatorname{sech}^{4}(q x)$ and

$$
\begin{aligned}
b(x, y)= & -\frac{1}{2} q^{4} \operatorname{sech}^{4}(q x) \operatorname{sech}^{4}(q y) e^{-q|x-y|} \\
& \times(\cosh [q(x+y)]-q|x-y|\{\cosh [q(x-y)] \\
& +\sinh [(q|x-y|)]\}) .
\end{aligned}
$$

According to the IST method the soliton amplitude $A$ and velocity $v$ are

$$
\begin{gathered}
A=-2 q^{2}+2 \Delta E \\
v=4 q^{2}-4 \Delta E
\end{gathered}
$$

Thus the corrections to the velocity and the absolute value of the amplitude $|A|$ are

$$
\begin{gathered}
\Delta v=4 \Delta E=\Delta_{1} v+\Delta_{2} v \\
\Delta A=-2 \Delta E=\Delta_{1} A+\Delta_{2} A
\end{gathered}
$$

where $\Delta_{1}$ and $\Delta_{2}$ denote the first- and second-order corrections in $\varepsilon(x)$, respectively. It is possible to represent the solution in the form of a single soliton plus weak radiation, when the perturbation expansion in (2.9) for the eigenvalue $E$ of the $L$ operator is valid. A crude condition for the validity of such a representation can be obtained by using the mean field method [11] and the condition is $\langle\Delta E\rangle /\langle E\rangle \ll 1$. It leads to the condition $\sigma^{2} q \ll 1$ for the case with multiplicative noise and the condition $\sigma^{2} Q^{2} / q^{3} \ll 1$ for the case with additive noise. The noise can also generate small-amplitude solitons embedded in a radiation field. This effect does not change the above estimate essentially.

\section{DISTRIBUTION FUNCTION}

Let us calculate the distribution function for the soliton parameters generated by the random initial pulses (2.2) and (2.3). To do this we use the cumulant method [12]. It follows from (2.9) that the corrections to the soliton parameters are the nonlinear functionals of the random process $\varepsilon(x)$. So the distribution function has a nonGaussian form. In accord with the cumulant method, the first and the second cumulant define the parameters of the Gaussian distribution function. In turn, the deviation from the Gaussian distribution function is described by the third- and other higher-order cumulants. The thirdorder cumulant describes the asymmetric distortion of the distribution function resulting in the distinction between the mean and most probable values of a random quantity.

For the calculation of cumulants we make use of the relations between cumulants and moments of a random quantity $\Delta[12,13]$

$$
\begin{aligned}
K_{1}= & M_{1} \simeq\left\langle\Delta_{2}\right\rangle+\left\langle\Delta_{4}\right\rangle+0\left(\varepsilon^{6}\right) \\
K_{2}= & M_{2}-M_{1}^{2} \simeq\left\langle\Delta_{1}^{2}\right\rangle+\left\langle\Delta_{2}^{2}\right\rangle \\
& -\left\langle\Delta_{2}\right\rangle^{2}+2\left\langle\Delta_{1} \Delta_{3}\right\rangle+O\left(\varepsilon^{6}\right) \\
K_{3}= & M_{3}-3 M_{1} M_{2}+2 M_{1}^{3} \\
\approx & 3\left\langle\Delta_{1}^{2} \Delta_{2}\right\rangle-3\left\langle\Delta_{2}\right\rangle\left\langle\Delta_{1}^{2}\right\rangle+O\left(\varepsilon^{6}\right) .
\end{aligned}
$$

Here $K_{n}$ and $M_{n}$ are the cumulants and moments of $n$th order, respectively. In expressions (3.1) all cumulants are written up to fourth order in $\varepsilon$ since $K_{3}$ is of order $\sim \varepsilon^{4}$. However, as it will be shown below, calculating the cumulants $K_{1}$ and $K_{2}$ up to order $\varepsilon^{2}$ is enough.

Using the formulas (2.9), (2.10), and (3.1) we obtain the following expressions for cumulants describing the statistical properties of the soliton amplitude for multiplicative noise initial conditions (2.2): 


$$
\begin{aligned}
K_{1} \approx & -2 \int_{-\infty}^{\infty} \int_{-\infty}^{\infty} d x d y B(x-y) b(x, y) \approx 1.2 q^{3} \sigma^{2} \\
K_{2} \approx & 4 \int_{-\infty}^{\infty} \int_{-\infty}^{\infty} d x d y B(x-y) a(x) a(y) \approx 3.7 q^{5} \sigma^{2}, \\
K_{3}= & +24 \int_{-\infty}^{\infty} \int_{-\infty}^{\infty} d x_{1} d y_{1} B\left(x_{1}-y_{1}\right) b\left(x_{1}, y_{1}\right) \int_{-\infty}^{\infty} \int_{-\infty}^{\infty} d x_{2} d y_{2} B\left(x_{2}-y_{2}\right) a\left(x_{2}\right) a\left(y_{2}\right) \\
& -24 \int_{-\infty}^{\infty} \int_{-\infty}^{\infty} \int_{-\infty}^{\infty} \int_{-\infty}^{\infty} d x_{1} d x_{2} d x_{3} d x_{4}\left\langle\varepsilon\left(x_{1}\right) \varepsilon\left(x_{2}\right) \varepsilon\left(x_{3}\right) \varepsilon\left(x_{4}\right)\right\rangle a\left(x_{1}\right) a\left(x_{2}\right) b\left(x_{3}, x_{4}\right) \\
\approx & 7.4 q^{8} \sigma^{4} .
\end{aligned}
$$

For simplicity we assume that the random process is $\delta$ correlated, i.e.,

$$
B(x-y)=\sigma^{2} \delta(x-y) .
$$

The cumulants describing the statistical properties of the soliton velocity are $K_{n}[v]=2^{n} K_{n}[A]$. It is worth noting that for the Gaussian random process $\varepsilon(x)$ the cumulant of fourth order $K_{4}$ is sixth order in $\varepsilon(x)$. We have calculated $K_{1}$ and $K_{2}$ up to order $\epsilon^{2}$ and $K_{3}$ up to order $\epsilon^{4}$. The explanation for this approximation will be given later. According to the cumulant method, the logarithm of the characteristic function of the random quantity, i.e., the logarithm of the Fourier transform of the distribution function, can be expanded in a power series. The coefficients of this series are the cumulants of the corresponding order. Making use of this representation we obtain the expression for the distribution function

$$
\begin{aligned}
P(\Delta A)= & \frac{1}{2 \pi} \int_{-\infty}^{\infty} d v \cos \left[v\left(\Delta A-K_{1}\right)+\frac{1}{6} K_{3} v^{3}\right] e^{-\frac{1}{2} K_{2} v^{2}} \\
\approx & \frac{1}{\sqrt{2 \pi K_{2}}} e^{-\frac{\left(\Delta A-K_{1}\right)^{2}}{2 K_{2}}}\left[1-\frac{K_{3}\left(\Delta A-K_{1}\right)}{2 K_{2}^{2}}\right. \\
& \left.+\frac{K_{3}\left(\Delta A-K_{1}\right)^{3}}{6 K_{2}^{3}}\right]
\end{aligned}
$$

In Fig. 1 we show $P(\Delta A)$ from Eq. (3.4) as function of $\Delta A$ for $q=1$ and $\sigma=0.1$ [Fig. 1(a)], $\sigma=0.5$ [Fig. $1(\mathrm{~b})$ ], and $\sigma=1.0$ [Fig. 1(c)] (solid curves). In order to show the distortion due to $K_{3}$ more clearly, the Gaussian distribution function with $K_{3}=0$ has been added (dashed curve). The asymmetric distortion due to $K_{3}$ is apparent. In the curve for $P(\Delta A)$ there exists a region with negative values of the distribution function. It is a well known peculiarity in the theory of cumulants arising from truncation of the cumulant series $[13,14]$. In our case this region is beyond the validity of the perturbation theory and its contribution to the statistical properties of the soliton parameters is negligible.

Using the expressions (3.4) we can calculate the statistical characteristics of the soliton amplitude and velocity. For example, the correction to the most probable (MP) value $(\Delta A)_{\text {MP }}$ of the soliton amplitude is

$$
(\Delta A)_{\mathrm{MP}}=K_{1}-\frac{K_{3}}{2 K_{2}} \approx 0.2 q^{3} \sigma^{2}
$$

However, the mean value $\langle\Delta A\rangle$ is

$$
\langle\Delta A\rangle=K_{1}=1.2 q^{3} \sigma^{2},
$$

i.e., 6 times larger. The difference between these two values is

$$
\langle\Delta A\rangle-(\Delta A)_{\mathrm{MP}}=K_{3} / 2 K_{2} \approx q^{3} \sigma^{2},
$$

i.e., it is proportional to $q^{3}$. It means that the asymmetric distortion of the distribution function is larger for solitons with larger amplitudes.

From Eq.(3.4) it is also seen that the cumulant $K_{3}$ appears only in the ratio $K_{3} / 2 K_{2}^{2}$. The combined contribution of $K_{2}$ and $K_{3}$ is of order $\varepsilon^{2}$. This circumstance proves the validity of calculating $K_{3}$ up to order $\varepsilon^{4}$ and $K_{1}$ and $K_{2}$ up to order $\varepsilon^{2}$. So the most probable value and the mean value of the soliton amplitude are

$$
\begin{aligned}
A_{\mathrm{MP}} & =-2 q^{2}\left(1+0.1 q \sigma^{2}\right), \\
\langle A\rangle & =-2 q^{2}\left(1+0.6 q \sigma^{2}\right),
\end{aligned}
$$

respectively. The analogous formulas are valid for the soliton velocity. Thus, from Eqs. (3.2c) and (3.4) it follows, that during propagation, part of the energy of the stochastic component is transferred to the soliton.

For the case of additive noise (2.3) we obtain by performing analagous calculations, the following expressions for the cumulants:

$$
\begin{aligned}
& K_{1}=\frac{\sigma^{2} Q^{2}}{q} a_{12}(\alpha), \\
& K_{2}=\sigma^{2} q Q^{2} a_{21}(\alpha)+\frac{\sigma^{4} Q^{4}}{q^{2}} a_{22}(\alpha), \\
& K_{3}=\sigma^{4} Q^{4} a_{32}(\alpha),
\end{aligned}
$$

where $\alpha=p / q$ and $l_{r}=1 / p$ is the soliton and noise lengths ratio. For $\alpha \ll 1$ we obtain the approximate expressions for $a_{i j}$

$$
\begin{array}{r}
a_{12}=0.25\left(2.66-5.72 \alpha^{2}\right), \quad a_{21}=0.5\left(1.66-0.86 \alpha^{2}\right), \\
(3.10 \mathrm{a}) \\
a_{22}=0.125\left(1.44-4.61 \alpha^{2}\right), \quad a_{32}=0.75\left(0.3+0.66 \alpha^{2}\right) .
\end{array}
$$

It is difficult to obtain analytical expressions for arbitrary 
values of $\alpha$. The $\alpha$-independent terms are the same for other noise models with finite duration. We have calculated numerically the $a_{i j}$. The results agree well with the expansions (3.10) and show that the parameter $a_{32}$ has a maximum at $\alpha=0.6$. The other functions $a_{i j}$ decrease as $\alpha$ increases. It should be noted that for $p \rightarrow q$ and $Q=2 q^{2}$ the result (3.9) coincides with Eq. (3.2). The mean correction to the amplitude is

(a)
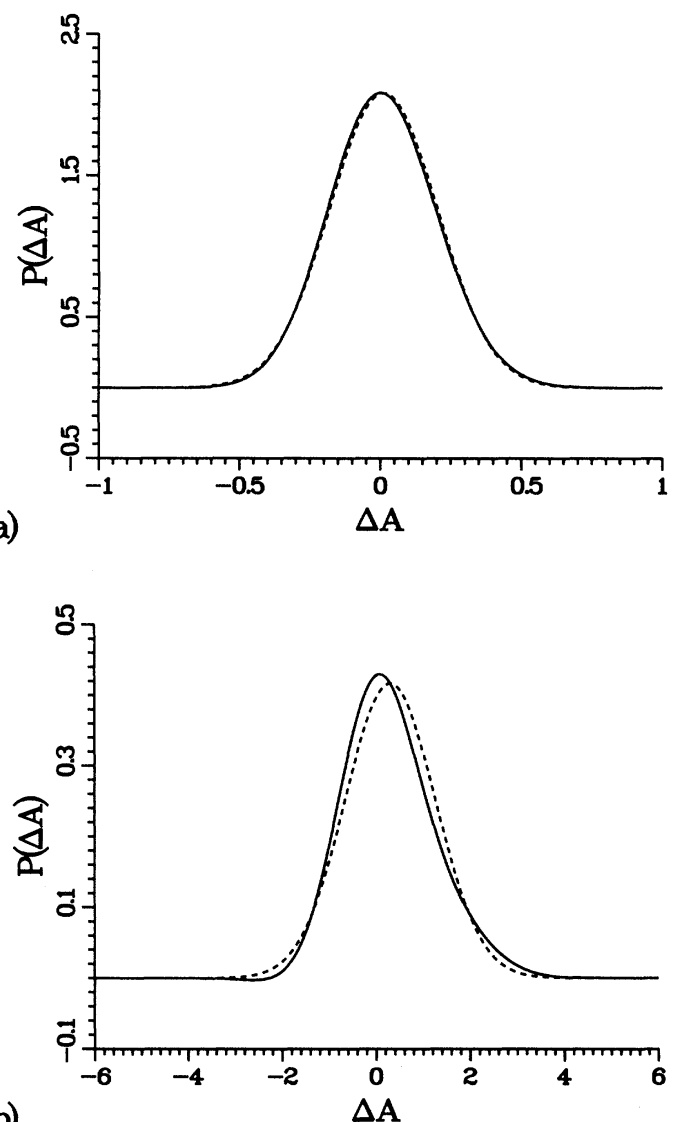

(b)

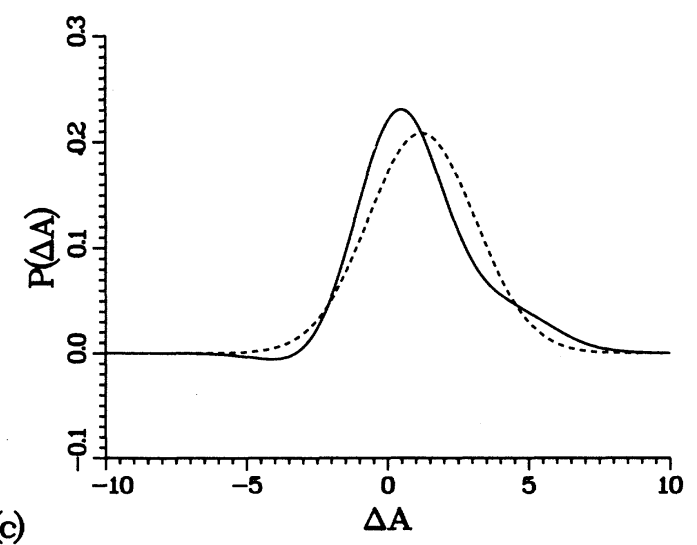

FIG. 1. Graphical display of the distribution function in Eq. (3.4) for $q=1$ (solid curves). (a) $\sigma=0.1$, (b) $\sigma=0.5$, and (c) $\sigma=1.0$. For comparison the Gaussian distribution function $\left(K_{3}=0\right)$ is added as the dashed curve.

$$
\langle\Delta A\rangle=\frac{2 \sigma^{2} Q^{2}}{3 q}
$$

The inverse dependence on $q$ is natural because, as the soliton amplitude is increased, the influence of the additive noise is reduced. This is in contradiction to the multiplicative noise case (3.8). The most probable amplitude correction is

$$
(\Delta A)_{\mathrm{MP}}=0.53 \frac{\sigma^{2} Q^{2}}{q} .
$$

It follows that the orders of magnitude of $\langle\Delta A\rangle$ and $(\Delta A)_{\text {MP }}$ are the same. In distinction from the multiplicative noise case we have

$$
A_{\mathrm{MP}}=-2 q^{2}\left(1+0.25 \frac{\sigma^{2} Q^{2}}{q}\right),
$$

i.e., $A_{\mathrm{MP}}$ is increasing and $\langle A\rangle$

$$
\langle A\rangle=-2 q^{2}\left(1+\frac{\sigma^{2} Q^{2}}{3 q^{3}}\right) .
$$

As seen from (3.14) the absolute value of the mean amplitude increases in comparison with the amplitude $2 q^{2}$. The coefficient characterizing the deviation of the distribution function $P(A)$ from the Gaussian form is $\gamma_{3}=$ $K_{3} / K_{2}^{3 / 2}$ and it is proportional to $\sqrt{c}$, where $c=\sigma^{2} q$ for multiplicative noise and $c=\sigma^{2} Q^{2} / q^{3}$ in the case of additive noise. So we can conclude that in the case of multiplicative noise the asymmetry is larger in comparison with additive noise. When $l_{r} \rightarrow l_{s}$, the asymmetry grows. The influence of the noise on the soliton parameters is stronger when the widths of the noise and soliton are of the same order.

\section{NUMERICAL SIMULATIONS}

The KdV equation (2.1) has been solved numerically by means of the Fourier split step method $[15,16]$ in the interval $x \in[-L ; L], L=40$, with periodic boundary conditions. The number of discretization points in the $x$ direction is 1024 and with $L=40$ this leads to a space discretization step $d x$ of size $d x=0.0781$. The random initial condition (2.2) results in small-amplitude radiation modes that propagate away from the back part of the emerging single soliton. Due to the periodic boundary condition, those modes will run out at $x=-L$ and reenter at $x=+L$, propagating toward the soliton front. As we need to find the amplitude of the undisturbed emerging soliton, we must avoid collision between the reentering modes and the soliton. Therefore, we have inserted an absorbing boundary at $x= \pm L$ in order to destroy the radiation modes. This is done by solving

$$
u_{t}-6 u u_{x}+u_{x x x}+\alpha(x) u=0,
$$

where the function $\alpha(x)$ is localized at the boundaries and otherwise chosen rather arbitrarily as 


$$
\alpha(x)=A_{d} \operatorname{sech}\left(\frac{x \pm L}{w}\right)
$$

In the computations we have chosen $A_{d}=500$ and $w=$ 0.4 throughout.

In the Fourier split step method, Eq. (4.1) is divided into its nonlinear and linear parts according to

$$
\begin{aligned}
& u_{t}=6 u u_{x}+\alpha(x) u \\
& u_{t}=-u_{x x x}
\end{aligned}
$$

The solution is advanced in small time steps $d t$ by solving Eqs. (4.3a) and (4.3b) in turn. Assuming $u_{x}$ to be constant during the short time interval $[t ; t+d t]$, we advance Eq. (4.3a) according to

$$
u(x, t+d t)=u(x, t) \exp \left[\left(6 u_{x}+\alpha(x)\right) d t\right] .
$$

The space derivative of $u$ is determined numerically using Fourier transforms. The linear part $(4.3 \mathrm{~b})$ can be solved analytically, thereby advancing the solution according to

$$
u(x, t+d t)=F^{-1}\left\{F[u(z, t)] \exp \left(i k^{3} d t\right)\right\},
$$

where $F$ denotes the Fourier transform with respect to $x$ and $F^{-1}$ its inverse. The wave number is designated by $k$.

In the initial conditions (2.2) we have used Gaussian distributed random numbers generated by a standard subroutine. In order to take proper care of the discretization in the space variable $x$, we must divide the Gaussian random numbers by $\sqrt{d x}, d x$ being the space discretization step, before inserting into the initial condition [17].

Figure 2 shows the evolution of one realization of the initial condition in Eq. (2.2) using $q=1$ and $\sigma=0.1$. The small-amplitude oscillatory waves rapidly propagates away from the back of the emerging single soliton. At time $t \approx 1$ the soliton has clearly separated from the small-amplitude waves, allowing for the measurement of its amplitude. In the statistical treatment we have cho-

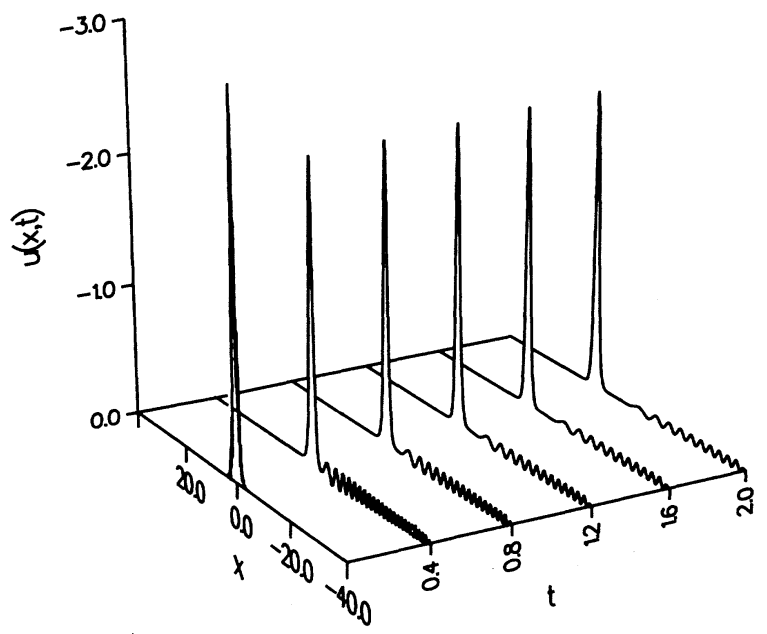

FIG. 2. Evolution of a random modulated soliton [Eq. (2.2)] for $q=1$ and $\sigma=0.1$. sen to propagate the soliton until $t=2$, before measuring its amplitude.

In Fig. 3(a) we depict the statistical results of $N=$ 2000 realizations of the initial condition (2.2) propagated from $t=0$ until $t=2$ for $q=1$ and $\sigma=0.1$. The solid curve is a histogram counting the number of initial conditions leading to amplitude deviations $\Delta A$ between $\Delta A_{n} \leq \Delta A<\Delta A_{n}+d A$ with $d A=0.04$ and $\Delta A_{n}=n d A, n$ being an integer. The curve is normalized by the factor $1 /(N d A)$. Figure $3(\mathrm{~b})$ shows the associated histogram for $\sigma=0.5$, using $N=1197$ realizations and with $d A=0.3$. The numerical results are compared with the result from Eq. (3.4) (dashed curve). The theoretical prediction fits well the statistical estimate from the simulations. For $\sigma=0.1$ [Fig. 3(a)] the variance is so small that the asymmetric distribution is barely observed. However, in the case of $\sigma=0.5$ [Fig. 3(b)] the asymmetry is clearly observed, confirming the redistribution of energy from the linear modes to the soliton.
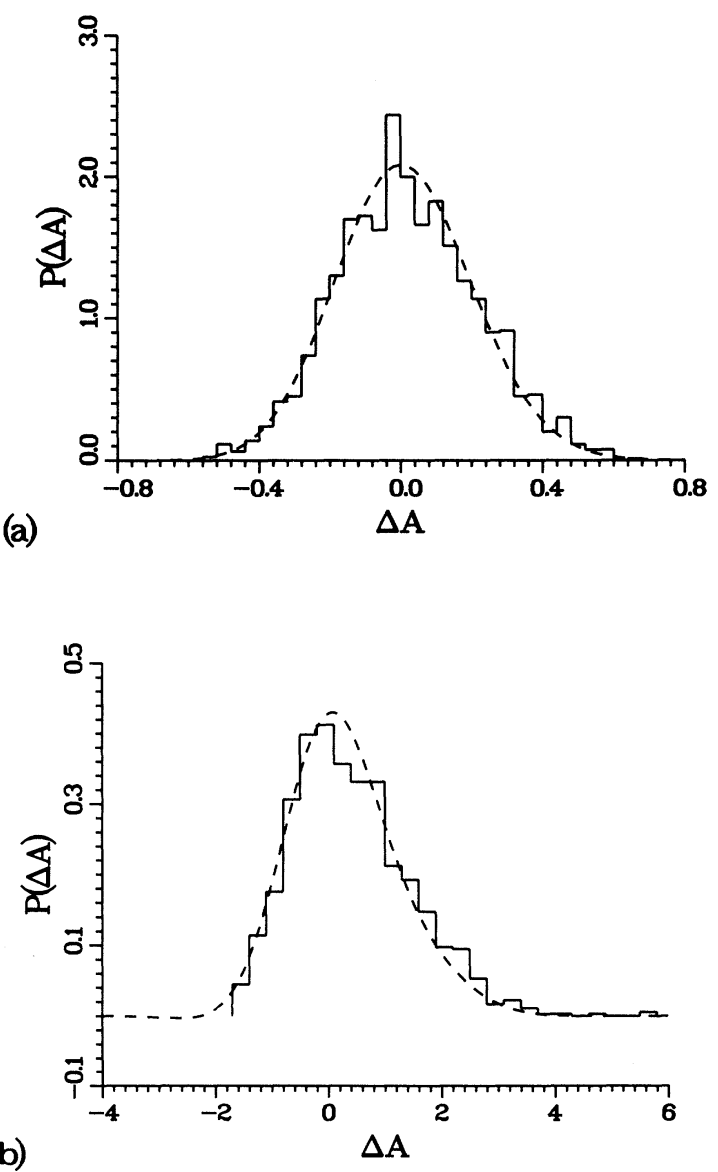

FIG. 3. Histograms of the probability distribution of the amplitude deviation $\Delta A$ obtained from numerical simulations of $N=2000$ samples using $q=1$. (a) $\sigma=0.1$ and (b) $\sigma=0.5$. The dashed curves show the theoretical calculated distribution of $\Delta A$ from Eq. (3.4). 


\section{APPLICATION TO THE RANDOMLY MODULATED SOLITONS PROPAGATION IN A NONLINEAR TRANSMISSION LINE AND IN AN OPTICAL FIBER}

Here we will consider two physical applications of the results obtained for multiplicative and additive noises.

(i) As an example of the multiplicative noise effect on a soliton, let us consider the propagation of electromagnetic solitons excited by a noncoherent source in a nonlinear $L C$-transmission line. The line is represented by the $L C$-cell chain, with the nonlinear capacitance $C(V)=C_{0}-C_{N} V$, where $V$ is the voltage. We can derive the $\mathrm{KdV}$ equation in the form (2.1) for the dimensionless voltage $u=-V / V_{0}, V_{0}=C_{0} / C_{N}$, where the dimensionless variables are introduced

$$
t=\frac{1}{3 \omega_{0}} T, \quad \omega_{0}=\frac{1}{\sqrt{L C_{0}}}, \quad x=\frac{2\left(X-a \omega_{0} T\right)}{a} .
$$

Here $a$ is the $L C$ cell size. The solitonic solution in the physical variables has the form

$$
V_{s}(X, T)=A_{s} \operatorname{sech}^{2}\left(\frac{X-v_{s} T}{L_{s}}\right), \quad A_{s}=\frac{2 q^{2} C_{0}}{C_{N}} .
$$

It follows from (3.14) and (5.2) that $\left\langle\Delta v_{s}\right\rangle=0.4 q^{3} \sigma^{2} v_{0}$, where $v_{0}$ is the unperturbed soliton velocity in the line. If we take $C_{0}=700 \mathrm{pF}, C_{N}=140 \mathrm{pF} / \mathrm{V}, L=6.8 \mathrm{mkG}$, $a=2 \mathrm{~cm}, v_{0}=2.86 \times 10^{7} \mathrm{~cm} / \mathrm{sec}$ [18], and $\sigma^{2}=0.1$, we have velocity fluctuations of order $10^{6} \mathrm{~cm} / \mathrm{sec}$.

(ii) The results obtained above for the additive noise case has important applications to the problem of the randomly modulated dark soliton propagation in optical fibers. The random modulation can be induced, for example, by the amplifier noise in fibers. As is known, the small-amplitude dark soliton in optical fibers can be described as a KdV soliton [19]. Indeed, the pulse propagation in optical fibers in the region of positive group dispersion $\left(K_{\omega \omega}^{\prime \prime}>0\right)$ is described by the nonlinear Schrödinger equation

$$
i u_{x}-u_{t t}+2|u|^{2} u=0 .
$$

The dark soliton solution has the form

$$
\begin{aligned}
u_{s} & =u_{0}\left[(\lambda-i \nu)^{2}+\exp (z)\right][1+\exp (z)]^{-1} e^{2 i u_{0}^{2} x} \\
z & =2 \nu u_{0}\left(t-t_{0}-2 \lambda u_{0} x\right), \lambda^{2}=1-\nu^{2}
\end{aligned}
$$

where $u_{0}$ is the amplitude of background and $\nu$ is the amplitude of the dark soliton. When $\nu^{2} \ll 1$, i.e., in the small-amplitude dark soliton case, we obtain

$$
u_{s} \approx u_{0}\left[1-\frac{1}{2} \nu^{2} \operatorname{sech}^{2}(z / 2)\right] \exp \left[2 i u_{0}^{2} x+i \varphi(x, t)\right],
$$

where $\varphi(x, t)=-2 \nu /\left(1+e^{z}\right)$ and $z=2 \nu u_{0}\left(t \mp 2 u_{0} x \pm\right.$ $\left.u_{0} \nu^{2} x\right)$. Using the representation

$$
u=u_{0}[1+4 \nu(x, t)] e^{2 i u_{0}^{2} x+i \varphi(x, t)}
$$

the new variables

$$
\tau=\varepsilon\left(t-4 u_{0} x\right), \quad y=-4 u_{0} \varepsilon^{3} x, \quad \varepsilon \kappa=\nu u_{0},
$$

and the expansion

$$
\begin{aligned}
& \nu=\varepsilon^{2} \nu_{0}+\varepsilon^{4} \nu_{1}+\cdots, \\
& \varphi=\varepsilon \varphi_{0}+\varepsilon^{3} \varphi_{1}+\cdots,
\end{aligned}
$$

we obtain the $\mathrm{KdV}$ equation for $\nu_{0}$

$$
\nu_{0 y}-6 \nu_{0} \nu_{0 \tau}+\nu_{0 \tau \tau \tau}=0
$$

with the soliton solution in the form

$$
\nu_{0}(y, \tau)=-2 \kappa^{2} \operatorname{sech}^{2} \kappa\left(\tau-4 \kappa^{2} y\right)
$$

For the randomly modulated initial soliton (2.2) we obtain, using the formula (3.6), the expression for the mean value of correction to amplitude of the dark soliton

$$
\left\langle\Delta\left(2 \kappa^{2}\right)\right\rangle=\frac{2 \sigma^{2} Q^{2}}{3 \kappa}
$$

Because the velocity of the dark soliton is a random parameter, so is the arrival time on receivers. For this reason there is a finite probability for simultanious arrival of two solitons on the receiver. This leads to a loss of transmitted information, which is defined by the width of the soliton velocity distribution function [20]. It should be noted that in the work in Ref. [21], the influence of infinite length additive amplifier noise on dark solitons of large amplitude has been studied. In the case of $\alpha \rightarrow 0$ our results qualitatively agree with the results of [21].

\section{CONCLUSION}

In conclusion, we review briefly the results achieved in this work. We have derived the distribution function for the parameters of $\mathrm{KdV}$ solitons generated from stochastic initial conditions. This distribution function has non-Gaussian form. We calculate the mean and the most probable values of the formed soliton amplitudes. The peak of the distribution function for the amplitude deviation $\Delta A$ is at the point $K_{1}=2 \sigma^{2} Q^{2} / 3 q$ (additive noise) and at $K_{1}=1.2 q^{3} \sigma^{2}$ (multiplicative noise). The numerical modeling of the $\mathrm{KdV}$ equation with the random initial conditions demonstrates good agreement with the analytical results. The values of the asymmetry coefficient are larger for higher soliton amplitudes in the case of multiplicative noise and inversely proportional to the soliton amplitude for additive noise perturbation. We can conclude that during propagation in the medium, part of the energy of the stochastic component is transferred to the soliton. The results obtained are applied to the evolution of randomly modulated dark solitons in optical fibers and electromagnetic solitons in the nonlinear transmission lines. It should be noted that the 
problems concerning the evolution in the case of colored noise and large (multisoliton) random initial conditions are still open (some results on this matter were obtained in [4]). A second class of problems is represented by the evolution of random initial conditions in the systems described by the nearly integrable nonlinear equations. This problem will be considered separately.

\section{ACKNOWLEDGMENTS}

F.Kh.A. is grateful to IMM and MIDIT, DTU for financial support. This work is partially supported by the International Science Foundation (Grant No. MZG000) and the Danish Research Council through Contract No. 5210551-1 and No. 9313393 (supercomputing projects).
[1] F.Kh. Abdullaev, S.A. Darmanyan, and P.K. Khabibullaev, Optical Solitons (Fan, Tashkent, 1987).

[2] J.N. Elgin, Phys. Lett. 110A, 441 (1985).

[3] V.V. Konotop and L.S. Vazquez, Nonlinear Random Waves (World Scientific, Singapore, 1994).

[4] F.Kh. Abdullaev and S.A. Darmanyan, Zh. Tekh. Fiz. 58, 265 (1988) [Sov. J. Tech. Phys. 33, 162 (1988)].

[5] V.E. Zakharov, S.A. Manakov, S.P. Novikov, and L.P. Pitaevsky, Theory of Solitons (Nauka, Moscow, 1980).

[6] S.N. Gurbatov, S. Malakhov, and A.I. Saichev, Nonlinear Random Waves and Turbulence in Nondispersive Media: Waves, Rays, Particles (Manchester University Press, Manchester, 1991).

[7] F.G. Bass, Yu. S. Kivshar, and V.V. Konotop (unpublished).

[8] A.I. D'yachenko et al., Zh. Eksp. Teor. Fiz. 96, 2026 (1989) [Sov. Phys. JETP 69, 1144 (1989)].

[9] F.Kh. Abdullaev and S.A. Darmanyan, Radiofizika 32, 1048 (1989).

[10] D. Krokel, N.J. Hales, G. Ginliani, and D. Grishkovsky, Phys. Rev. Lett. 60, 29 (1988).
[11] J. Ziman, Models of Disorder (Cambridge University Press, Cambridge, 1979).

[12] V.I. Klyatzkin, Stochastic Differential Equations and Waves in Random Media (Nauka, Moscow, 1984).

[13] S. Malakhov, Nongaussian Random Processes and Cumulant Analysis (Nauka, Moscow, 1985).

[14] H. Risken, The Fokker-Planck Equation (SpringerVerlag, Heidelberg, 1984).

[15] R.H. Hardin and F.D. Tappert (unpublished). SIAM Rev. Chron. 15, 423 (1973).

[16] T.R. Taha and M.J. Ablowitz, J. Comput. Phys. 55, 203 (1984).

[17] R. Badii, T. Schneider, and M.P. Soerensen, Phys. Rev. B 35, 297 (1987).

[18] K.E. Longren, in Soliton in Action, edited by K.E. Longren and A.C. Scott (Academic, New York, 1978).

[19] Yu. S. Kivshar, IEEE J. Quantum Electron 29, NI, 250 (1993).

[20] J.P. Gordon and H.A. Haus, Opt. Lett. 11, 665 (1994).

[21] Yu. S. Kivshar, H. Halterman, Ph. Emplit, and J.P. Hamaide, Opt. Lett. 19, 19 (1994). 